\title{
Training in ERCP: a multifaceted enterprise now more than ever
}

\section{Dear Editor:}

We read with great interest the recent article by Frost et al. examining whether trainee involvement in endoscopic retrograde cholangiopancreatography (ERCP) is detrimental to cannulation success [1]. ERCP training has evolved considerably over the last 15 years, and the definition of competency in ERCP has expanded well beyond the ability to cannulate a duct of interest to incorporate clinical decision-making and imaging interpretation. Contemporaneously, clinical demands (e.g. decreases in diagnostic ERCP case volume, need to minimize procedural duration, increasing concerns regarding safety, and relative value unitbased compensation models) have continued to place ERCP training at a premium [2]. Therefore, the study by Frost et al. is timely and relevant.

The study prospectively followed 2 senior consultant endoscopists and their 3 trainees (all with $<50$ prior ERCP experience) over an 18-month period in a United Kingdom hospital with a caseload of approximately 330 ERCPs/year. Presence of a trainee was not randomized but rather "pragmatic," and only native papillae were included. Once the duodenal papilla (major or minor, depending on the particular) was reached and stable scope position attained, 6 minutes were allotted for cannulation; if achieved, the trainee was allowed to continue, otherwise the supervising consultant would resume. A total of 219 ERCPs were analyzed (134 with trainee, 85 without); procedure difficulty was $61 \%$ grade $1,37 \%$ grade 2 , and $2 \%$ grade 3 [3]. The central findings were that: 1) cannulation success was similar with or without a trainee present (91\% vs. 93\%, $P=0.8$ ); and 2 ) mean time to biliary cannulation with a trainee present was 7 minutes, compared to 5 minutes without trainee. No serious adverse events were reported. The investigators' main conclusion was that with their ERCP training protocol, trainee involvement in routine secondary care
ERCP does not decrease cannulation success.

The study and its aims are commendable; however, several questions should be considered when interpreting the findings and potentially applying them broadly: 1) Were the two groups balanced (e.g. with regard to patient age, gender, and location and appearance of the papilla); 2) Could "pragmatic" determination of trainee participation have led to triaging of complex or challenging cases to consultant only; 3 ) Is the 6-minute rule used in the training protocol irrespective of number of "touches" or patient-level (e.g. anatomical) factors? Previous research has found that 10 minutes may be most appropriate for trainees to attempt cannulation [4], thus the basis for the 6-minute interval is curious; 4) At what number of ERCPs, on average, did the trainees in the investigators' training protocol achieve competency; and 5) There were no cases of "complicated pancreatitis" out of the 219 ERCPs included in the study; did any patients require hospitalization or additional investigation for pancreatitis (but no "further intervention" per se)? Having no cases of post-ERCP pancreatitis out of 219 ERCPs represents a markedly low incidence.

Answers to the aforementioned questions could greatly enhance interpretation and generalizability of the study findings. In addition, propensity scorebased sensitivity analysis or other statistical methods (e.g. multivariate logistic regression) could be implemented to help strengthen the study and its findings [5]. Ultimately, a larger, well-designed randomized trial would best evaluate the impact of trainee involvement and delineate optimal ERCP training protocols.

\section{Competing interests}

The authors

James H. Tabibian ${ }^{1}$, Joseph W. Leung ${ }^{2}$

1 Division of Gastroenterology, Department of Medicine Olive View-UCLA Medical Center, Sylmar, CA, United States

2 Division of Gastroenterology and Hepatology, UC Davis Medical Center, Sacramento, CA, United States

\section{Corresponding author}

\section{Dr. James H. Tabibian}

Department of Medicine, 14445 Olive View

Dr., 2B-182, Sylmar, CA 91203

Fax: +1-747-210-4573

jtabibian@dhs.lacounty.gov

\section{References}

[1] Frost JW, Kurup A, Shetty S et al. Does the presence of a trainee compromise success of biliary cannulation at ERCP? Endosc Int Open 2017; 5: E559-E562

[2] Leung JW, Chung RS. Training in ERCP. Gastrointest Endosc 1992; 38: 517 - 518

[3] Cotton PB, Eisen G, Romagnuolo J et al. Grading the complexity of endoscopic procedures: results of an ASGE working party. Gastrointest Endosc 2011; 73: 868 874

[4] Pan Y, Zhao L, Leung J et al. Appropriate time for selective biliary cannulation by trainees during ERCP - a randomized trial. Endoscopy 2015; 47: 688-695

[5] Li L, Shen C, Wu AC et al. Propensity scorebased sensitivity analysis method for uncontrolled confounding. Am J Epidemiol 2011; 174: $345-353$

\section{Bibliography}

DOI http://dx.doi.org/10.1055/s-0043-121003

Endoscopy International Open 2018; 06: E90

(c) Georg Thieme Verlag KG

Stuttgart · New York

ISSN 2364-3722 